\title{
Connectome-Wide Phenotypical and Genotypical Associations in Focal Dystonia
}

\author{
Stefan Fuertinger ${ }^{1,2}$ and ${ }^{-K}$ Kristina Simonyan ${ }^{1}$ \\ ${ }^{1}$ Department of Neurology, Icahn School of Medicine at Mount Sinai, New York, New York 10029, and ${ }^{2}$ Ernst Strüngmann Institute (ESI) for Neuroscience \\ in Cooperation with Max Planck Society, Frankfurt, Germany 60528
}

Isolated focal dystonia is a debilitating movement disorder of unknown pathophysiology. Early studies in focal dystonias have pointed to segregated changes in brain activity and connectivity. Only recently has the notion that dystonia pathophysiology may lie in abnormalities of large-scale brain networks appeared in the literature. Here, we outline a novel concept of functional connectome-wide alterations that are linked to dystonia phenotype and genotype. Using a neural community detection strategy and graph theoretical analysis of functional MRI data in human patients with the laryngeal form of dystonia (LD) and healthy controls (both males and females), we identified an abnormally widespread hub formation in LD, which particularly affected the primary sensorimotor and parietal cortices and thalamus. Left thalamic regions formed a delineated functional community that highlighted differences in network topology between LD patients with and without family history of dystonia. Conversely, marked differences in the topological organization of parietal regions were found between phenotypically different forms of LD. The interface between sporadic genotype and adductor phenotype of LD yielded four functional communities that were primarily governed by intramodular hub regions. Conversely, the interface between familial genotype and abductor phenotype was associated with numerous long-range hub nodes and an abnormal integration of left thalamus and basal ganglia. Our findings provide the first comprehensive atlas of functional topology across different phenotypes and genotypes of focal dystonia. As such, this study constitutes an important step toward defining dystonia as a large-scale network disorder, understanding its causative pathophysiology, and identifying disorder-specific markers.

Key words: dystonia; functional connectome; genotype-phenotype interactions; spasmodic dysphonia

\section{Significance Statement}

The architecture of the functional connectome in focal dystonia was analyzed in a large population of patients with laryngeal dystonia. Breaking with the empirical concept of dystonia as a basal ganglia disorder, we discovered large-scale alterations of neural communities that are significantly influenced by the disorder's clinical phenotype and genotype.

\section{Introduction}

Laryngeal dystonia (LD), or spasmodic dysphonia, is an isolated task-specific focal dystonia that selectively affects the production of speech due to impaired voluntary control of vocal fold movements caused by involuntary spasms in the laryngeal muscles. As with other

\footnotetext{
Received Feb. 9, 2017; revised May 31, 2017; accepted June 7, 2017.

Author contributions: K.S. designed research; K.S. performed research; S.F. and K.S. analyzed data; S.F. and K.S. wrote the paper.

This work was supported by the National Institute on Deafness and Other Communication Disorders-National Institutes of Health (Grants R01DC01805 and R01NS088160 to K.S.). We thank Amanda Pechman, Melissa Choy, lan Farwell, Estee Rubien-Thomas, and Heather Alexander for help with subject recruitment and imaging data acquisition; Andrew Blitzer, Miodrag Velickovic, and Steven Fruch for patient referrals, Laurie Ozelius for initial genetic testing, and Giovanni Battistella for help with image processing.

The authors declare no competing financial interests.

Correspondence should be addressed to Kristina Simonyan, M.D., Ph.D., Department of Otolaryngology, Massachusetts Eye and Ear Infirmary, Harvard Medical School, 243 Charles St., Boston, MA 02114. E-mail: dystonia.research@gmail.com.

DOI:10.1523/JNEUROSCI.0384-17.2017

Copyright (C) 2017 the authors $\quad 0270-6474 / 17 / 377438-12 \$ 15.00 / 0$
}

focal dystonias, the exact causative pathophysiology of this debilitating movement disorder remains unclear. However, several neuroimaging studies have demonstrated disordercharacteristic functional and structural abnormalities in the laryngeal/orofacial sensorimotor and parietal cortices, basal ganglia, thalamus, and cerebellum, as well as altered striatal dopaminergic and GABAergic function in LD patients compared with healthy individuals (Haslinger et al., 2005; Ali et al., 2006; Simonyan et al., 2008; Simonyan and Ludlow, 2010, 2012; Simonyan et al., 2013; Samargia et al., 2016). More recent reports of the presence of dystonia in up to $16 \%$ of patient families (Kirke et al., 2015), threefold penetrance of dystonia mediational endophenotype in familial compared with sporadic LD cases (Termsarasab et al., 2016), and the association of brain abnormalities with gene mutations (i.e., DYT 25) and the polygenic risk (Putzel et al., 2016a, 2016b) have laid the foundation for a consideration of the interplay between potential etiological genetic factors and pathophysiological mechanisms 
that may lead to distinct clinical phenotypes and putative genotypes of LD.

Previously, we described LD phenotype- and genotype-specific alterations of functional activity within the sensorimotor and frontoparietal networks, specifically involving primary sensorimotor, premotor, and inferior parietal cortices (Battistella et al., 2016). LD phenotype-specific structural abnormalities have been reported in the primary and associative motor regions, whereas genotype-related structural changes have been found in the brain regions regulating phonological and sensory processing (Bianchi et al., 2017). Recent experimental evidence has also suggested that focal dystonias in general, including LD, may represent a disorder of large-scale functional networks as opposed to a basal ganglia pathology alone (Battistella et al., 2017).

In support of this emerging multifaceted and novel view on the causative pathophysiology of LD, we conducted detailed qualitative and quantitative investigations of the architecture of large-scale functional brain networks in a uniquely large population of LD patients compared with healthy subjects. Taking into account the potential for distinct pathophysiological mechanisms underlying different phenotypes and genotypes of this disorder, we examined the large-scale network topology across LD genotypes (sporadic and familial forms) and its clinical phenotypes (adductor and abductor forms). Based on the clinical history of the disorder, a patient without any familial history of $\mathrm{LD}$ and/or other isolated dystonias was considered to have the sporadic form, whereas a patient with at least one other blood relative affected with $\mathrm{LD}$ and/or other isolated dystonias was considered to have familial LD. Phenotype classifications were based on a patient's clinical diagnosis: adductor LD (ADLD) was characterized by involuntary vocal fold closure causing voice breaks and strained voice quality on vowels, whereas abductor LD (ABLD) showed intermittent breathy voice breaks extending voiceless consonants.

Using a graph theoretical approach (Bullmore and Sporns, 2009), we quantified large-scale network architecture by investigating the influence of a single region on network communication pathways (i.e., local network features) and the formation of neural communities of dense regional connectivity profiles (i.e., global network features). This approach allowed us to assess regional and network-wide aberrations related to LD, permitting a detailed investigation of whole-brain dystonic activity not limited to regions traditionally focused on in studies of LD pathophysiology such as basal ganglia and cerebellum. Based on previous knowledge, we hypothesized that the functional embedding of central motor control areas would be abnormally weak in LD accompanied by an overrepresentation of basal ganglia and thalamus within the network. We further hypothesized that our approach to a tiered, bottom-up analysis of network differences in the functional footprint of LD phenotypes and genotypes would allow us to identify genotype- and phenotype-specific differences in the dystonic cascade, which may ultimately underlie LD pathophysiology.

\section{Materials and Methods}

\section{Subjects}

A total of $90 \mathrm{LD}$ patients ( $54.5 \pm 12.9$ years; 73 females $/ 17$ males) and 32 healthy individuals ( $50.5 \pm 10.2$ years; 21 females $/ 2$ males) participated in this study (see detailed demographics in Table 1). All subjects were right-handed, native English speakers and none had any history of neurological (other than LD in the patient group), psychiatric, or laryngeal problems. All participants underwent neuroradiological evaluation, which found normal brain anatomy. Those LD patients who received botulinum toxin injections as part of their clinical symptom manage-
Table 1. Participant demographics

\begin{tabular}{|c|c|c|c|c|c|}
\hline & \multicolumn{2}{|l|}{ Sporadic } & \multicolumn{2}{|l|}{ Familial } & \multirow[b]{2}{*}{ HV } \\
\hline & ADLD & ABLD & ADLD & ABLD & \\
\hline Number of subjects & 32 & 31 & 20 & 7 & 33 \\
\hline Age (y; mean \pm SD) & $55.1 \pm 11.2$ & $52.2 \pm 12.5$ & $57.3 \pm 15.0$ & $54.6 \pm 15.8$ & $50.5 \pm 10.2$ \\
\hline Gender (female/male) & $24 / 8$ & $27 / 4$ & $17 / 3$ & $5 / 2$ & $21 / 12$ \\
\hline $\begin{array}{l}\text { Handedness (Edinburgh } \\
\text { Inventory) }\end{array}$ & & & Right & & \\
\hline Language & & Mono & lingual native $\mathrm{E}$ & English & \\
\hline $\begin{array}{l}\text { Disease duration } \\
\quad(y ; \text { mean } \pm S D)\end{array}$ & $12.8 \pm 8.9$ & $14.3 \pm 9.6$ & $20.7 \pm 14.5$ & $24.0 \pm 20.2$ & $\mathrm{~N} / \mathrm{A}$ \\
\hline
\end{tabular}

ment participated in the study at least 3 months after the last treatment to ensure that they were fully symptomatic at the time of study participation. Genetic screening confirmed that none of the participants were carriers of TOR1A (DYT1), THAP1 (DYT6), TUBB4A (DYT4), or GNAL (DYT25) mutations.

Patients and healthy individuals were assigned to experimental groups as follows. Group 1 was a comparison between the LD and normal states and included $32 \mathrm{LD}$ patients ( $49.8 \pm 11.1$ years; 22 females/ 10 males) and 33 healthy individuals ( $50.5 \pm 10.2$ years; 21 females $/ 12$ males). To allow for a balanced representation of different forms of $L D$, this patient group comprised 16 sporadic (8 ADLD/8 ABLD) and 16 familial LD cases ( 9 $\mathrm{ADLD} / 7 \mathrm{ABLD})$. Group 2 was a comparison between putative LD genotypes and included 27 sporadic LD patients without any past or present history of dystonia in family members $(57.6 \pm 13.0$ years; 22 females $/ 5$ males; $20 \mathrm{ADLD} / 7 \mathrm{ABLD}$ ) and 27 familial LD patients with a history of $\mathrm{LD}$ and/or other isolated dystonia in one or more family members (56.6 \pm 14.9 years; 22 females/5 males; 20 ADLD/7 ABLD). As a next step, to assess potential phenotype-related influences on LD genotype, we assembled more homogeneous patient groups with only the ADLD phenotype (20 sporadic ADLD: $58.25 \pm 11.7,17$ females/3 males vs 20 familial ADLD: $57.25 \pm 15.0$ years, 17 females $/ 3$ males). Because our study included only seven familial ABLD patients due to the extreme rarity of this particular form of the disorder, this cohort was not powered enough for a comparison with sporadic ABLD patients. This constituted a limitation of the current study, which should be addressed in a future study with a larger sample of familial ABLD patients. Group 3 was a comparison between different LD phenotypes and included 32 sporadic ADLD patients ( $55.1 \pm 11.2$ years; 24 females $/ 8$ males $)$ and 31 sporadic ABLD patients $(52.2 \pm 12.5$ years; 27 females $/ 4$ males). Due to the low number of familial ABLD patients, we did not compare this group with familial ADLD patients. However, we assessed the influence of putative genotype on ADLD and ABLD phenotypes by assembling more heterogeneous groups of mixed-genotype ADLD patients ( $55.1 \pm 12.7$ years; 29 females/10 males; 32 sporadic/7 familial ADLD) and mixed-genotype ABLD patients (52.6 \pm 13.0 years; 32 females/ 6 males; 31 sporadic/7 familial ABLD).

Written informed consent was obtained from all subjects before study participation and the study was approved by the Institutional Review Board of the Icahn School of Medicine at Mount Sinai. Some subjects participated in other studies conducted around the same time that assessed the resting-state and structural brain organization and neural correlates of abnormal sensory discrimination in LD (Battistella et al., 2016; Termsarasab et al., 2016; Bianchi et al., 2017).

\section{Experimental design}

Whole-brain functional images were acquired with a gradient-weighted echo-planar imaging (EPI) pulse sequence using an event-related sparsesampling design ( $\mathrm{TR}=2 \mathrm{~s}$ per volume and $8.6 \mathrm{~s}$ between volumes with total $\mathrm{TR}=10.6 \mathrm{~s}, \mathrm{TE}=30 \mathrm{~ms}, \mathrm{FA}=90, \mathrm{FOV}=240 \mathrm{~mm}$, voxel size $=$ $3.75 \times 3.75 \mathrm{~mm}, 36$ slices with $4 \mathrm{~mm}$ slice thickness). Four functional runs, including 24 sentence and 16 resting conditions, were acquired per subject. Subjects were instructed to listen to a meaningful grammatically correct English sentence presented via MR-compatible headphones within a 3.6-s interval, then repeat the same sentence within a 5-s period, after which a functional image was acquired within a 2-s interval while subjects silently fixated on a cross presented on a screen. Subjects listened to 10 sample sentences containing a high load of vowels (e.g., "Are the 
olives large?"; "Jack sat on a tack") to predominantly elicit ADLD symptoms and a high load of voiceless consonants (e.g., "He is hiding behind the house"; "Who says a mahogany highboy isn't heavy?") to predominantly elicit ABLD symptoms. All sentences were presented in a random order one at a time. Resting trials, during which the subjects were asked to silently fixate on a cross, were acquired as a baseline and pseudorandomized with the sentence production stimuli. To minimize motion artifacts, each subject's head was tightly cushioned within the coil, and all participants were monitored constantly for possible movements during the scanning session.

A high-resolution sagittal T1-weighted image was acquired using a magnetization prepared rapid acquisition gradient echo (MPRAGE) sequence $\left(172\right.$ contiguous slices, $1 \mathrm{~mm}^{3}$ voxel-size, $\mathrm{TR}=7.5 \mathrm{~ms}$, TE $=3.5 \mathrm{~ms}$, $\mathrm{FOV}=210 \mathrm{~mm}$ ) for registration of the functional images and to rule out any structural lesions.

Functional data analysis was performed using AFNI software (Cox, 1996) following a standard image preprocessing pipeline as described previously (Simonyan and Ludlow, 2012; Fuertinger et al., 2015; Termsarasab et al., 2016). Briefly, the first four volumes of all EPI datasets were removed to account for potential T1 stabilization effects and subsequently registered to the volume temporally closest to the high-resolution anatomical image. After spatial smoothing with a $4 \mathrm{~mm}$ Gaussian kernel, we performed multiple linear regression with the task regressor convolved with a canonical hemodynamic response function, six motion parameters (3D translation, pitch, roll, yaw rotation) to account for residual motion and three polynomials to control for low-frequency components such as scanner drift (Perrachione and Ghosh, 2013; Battistella et al., 2016; Termsarasab et al., 2016).

\section{Functional connectivity analysis: construction of functional networks}

To construct whole-brain functional networks of regional interactions, we used 212 regions of interest (ROIs) based on the cytoarchitectonic maximum probability maps and macrolabel atlas (Eickhoff et al., 2005; Fuertinger et al., 2015), including 142 cortical, 36 subcortical, and 34 cerebellar areas. In all subjects, pairwise interactions between regionally averaged time series during speech production were estimated by computing normalized mutual information (NMI) coefficients (Strehl and Ghosh, 2002; Fuertinger and Simonyan, 2016). The NMI was obtained by dividing the classic mutual information (Cover and Thomas, 1991) by the geometric mean of the associated Shannon entropies (Shannon, 1948), yielding a statistical dependence measure with values between zero (statistical independence) and one (mutual dependence). Therefore, in contrast to classic Pearson correlations, NMI edges are always non-negative, circumventing the need to account for negative weights in the computation of nodal influence metrics or to control for the emergence of negative cycles particularly affecting path-based measures (Fakcharoenphol and Rao, 2006). Because NMI coefficients reflect linear correlations and dependencies in higher moments (Brillinger, 2002), a nonzero Pearson correlation necessarily implies a positive NMI coefficient. Therefore, by definition, the nonzero structure of a pairwise Pearson correlation matrix is preserved in an NMI matrix, and NMI is not antagonistic but rather complementary to linear correlation (Kinney and Atwal, 2014).

In each subject, the constructed $212 \times 212$ NMI matrix was interpreted as the connectivity matrix of a weighted undirected graph, with ROIs representing the graph's nodes and NMI values serving as edge weights. The first estimate of each graph's connectivity profile was obtained by computing the network density, which was represented by the number of present to maximally possible edges in a graph. All subject networks were fully connected with a density of $100 \%$ and were thus subjected to thresholding (Fuertinger et al., 2015) following the rationale that very dense networks (graphs with a density $>50 \%$ ) tend to exhibit random graph characteristics (Humphries et al., 2006; Lynall et al., 2010). The density of the networks was reduced to $50 \%$ by removing edges with a weight less than an iteratively incremented percentage of the maximum weight in the graph (starting with $1 \%$ ) while simultaneously ensuring that the network did not fragment by fully disconnecting nodes.
After the thresholding of each individual graph, group-averaged networks were computed within each experimental group.

\section{Graph theoretical analysis and statistical analysis}

Network processing and visualization codes were written in Python using the open-source packages NumPy, SciPy (van der Walt et al., 2011), and Matplotlib (Hunter, 2007). Optimal modular decompositions were calculated in MATLAB (The MathWorks) using the Brian Connectivity Toolbox (Rubinov and Sporns, 2010). 3D renderings of the networks embedded in reference brain models were generated using the BrainNet Viewer (Xia et al., 2013).

Network metrics. Functional networks were analyzed to assess network segregation, integration, and nodal influence. Network segregation was estimated by computing the nodal clustering coefficient as the geometric mean of weights in triangles around each node (Onnela et al., 2005). In this manner, the presence of functional cliques in a node's local neighborhood was quantified. Network integration was approximated using the measure of global efficiency estimating a network's predilection for long-range communication (Sporns, 2011). Global efficiency was calculated as the average inverse shortest path length in the network (Latora and Marchiori, 2001) with connection lengths based on inverse edge weights. The influence of a single node within the graph was quantified by computing the nodal degree (i.e., the number of connected edges) and the nodal strength (i.e., the sum of connected edge weights; Rubinov and Sporns, 2010). Although the information provided by clustering coefficient, local efficiency, and modular organization of a network partially overlaps, the combination of these metrics provides a complete picture of local and global network properties (Sporns, 2011). The statistical significance of differences in network metrics across groups was assessed by using a two-tailed permutation $t$ test using 10,000 Monte Carlo randomizations at $p \leq 0.05$ adjusted for multiple comparisons by a $T_{\max }$ approach for the maximal test statistic (Pesarin, 2001).

Community-based network organization. To examine the global reconfiguration of functional networks in normal and diseased states, we performed a graph community analysis by estimating the optimal modular decomposition of a network, which divides a graph into nonoverlapping, connected groups of nodes (i.e., modules) with a maximal number of within-module edges and minimal number of between-module links (Fortunato, 2010; Fuertinger et al., 2015, 2016). A heuristic optimization strategy based on the Kernighan-Lin algorithm (Sun et al., 2009) was used to maximize the Newman modularity (Newman, 2006) to approximate each graph's optimal modular decomposition. An initial artificial community assignment, in which every node formed a module, was iteratively refined by solving the modularity maximization problem 100 times per network to account for the random nature of the used optimization algorithm, as described previously (Fuertinger et al., 2015). The final community affiliation was computed based on the average nodal module assignment, with node \#1 serving as a reference to account for random module numbering. As a first step to estimate the similarity of community structures between groups, we calculated their respective partition distances $p d$, which represented the normalized variation of information between the respective community affiliation vectors (Meila, 2007). In a follow-up analysis, we examined differences in spatial community structure and hub formation between the groups.

$H u b$ formation. Nodes exhibiting both degree and strength values at least $1 \mathrm{SD}$ larger than the respective average degree and strength in the network were defined as bivariate hubs. Nodes with both degree and strength values in the top $25 \%$ of the network but not satisfying the hub criterion were classified as high-influence nodes. To examine hub formation based on the number of connected intermodule vs intramodule edges, the computed optimal modular decomposition of each network was used to calculate the nodal participation coefficient $p c_{\mathrm{i}}(i=1, \ldots$, 212; van den Heuvel and Sporns, 2011; Fuertinger et al., 2015). In a network with $m \geq 2$ modules, the maximum value of $p c_{\mathrm{i}}$ is $1-1 / m$. If a hub exhibited a $p c_{\mathrm{i}}$ value within $10 \%$ of this maximum value, then it was classified as a connector hub linking two or more communities. If a hub showed a lower $p c_{\mathrm{i}}$ value, it was defined as a provincial hub connecting nodes within the same community. 
Table 2. Summary statistics of graph metrics in the group-averaged networks

\begin{tabular}{lllll}
\hline & \multicolumn{4}{l}{ Network metric } \\
\cline { 2 - 5 } & Degree & Strength & Clustering & Efficiency \\
\hline Controls & $106.52 \pm 36.07$ & $22.97 \pm 8.90$ & $0.14 \pm 0.01$ & $0.27 \pm 0.04$ \\
General LD & $105.60 \pm 40.40$ & $23.27 \pm 10.11$ & $0.15 \pm 0.02$ & $0.27 \pm 0.03$ \\
$p$ & 0.97 & 0.94 & $0.0001^{*}$ & 0.99 \\
Familial LD (ABLD + ADLD) & $106.44 \pm 37.23$ & $23.56 \pm 9.27$ & $0.14 \pm 0.02$ & $0.28 \pm 0.04$ \\
Sporadic LD (ABLD + ADLD) & $106.16 \pm 39.54$ & $23.02 \pm 9.70$ & $0.14 \pm 0.02$ & $0.27 \pm 0.02$ \\
$p$ & 0.99 & 0.79 & 0.85 & 0.23 \\
ADLD (Sporadic only) & $106.24 \pm 40.26$ & $22.64 \pm 9.71$ & $0.14 \pm 0.02$ & $0.26 \pm 0.03$ \\
ABLD (Sporadic only) & $105.54 \pm 39.89$ & $23.89 \pm 10.32$ & $0.15 \pm 0.01$ & $0.28 \pm 0.03$ \\
$p$ & 0.98 & 0.35 & $0.0001^{*}$ & 0.07 \\
Familial LD (ADLD only) & $105.78 \pm 36.33$ & $23.91 \pm 9.31$ & $0.14 \pm 0.02$ & $0.28 \pm 0.02$ \\
Sporadic LD (ADLD only) & $105.95 \pm 38.23$ & $23.40 \pm 9.62$ & $0.14 \pm 0.01$ & $0.27 \pm 0.03$ \\
$p$ & 0.99 & 0.82 & 0.91 & 0.21 \\
ADLD (Sporadic + familial) & $106.45 \pm 40.12$ & $22.70 \pm 9.66$ & $0.14 \pm 0.02$ & $0.27 \pm 0.03$ \\
ABLD (Sporadic + familial) & $105.87 \pm 40.25$ & $23.60 \pm 10.15$ & $0.15 \pm 0.02$ & $0.28 \pm 0.03$ \\
$p$ & 0.98 & 0.57 & $0.001^{*}$ & 0.16 \\
\hline
\end{tabular}

Degree, strength, and clustering coefficient values are given as averages (mean \pm SD) of nodal values across the respective group-averaged network. Efficiency was estimated by averaging the global efficiency of per-subject networks in the respective group.

*Statistically significant differences in the associated network metrics between the analyzed groups at $p \leq 0.05$ adjusted for multiple comparisons using a single-step correction of the maximal test statistic in a 2-tailed permutation test.

\section{Results}

\section{Group 1: overall characterization of the LD functional connectome}

Comparisons of functional networks between healthy individuals and LD patients (including ADLD, ABLD, sporadic, and familial forms) showed significantly increased network segregation (estimated by the clustering coefficient) in the patient group (controls: $0.14 \pm 0.02$; LD: $0.15 \pm 0.02, p<1 e-3$ ) but similar network integration (estimated by the global efficiency) between the two groups (controls: $0.27 \pm 0.04$; LD: $0.27 \pm 0.03, p=0.9$ ). Nodal influence quantified by degree and strength failed to exhibit pronounced differences between the healthy and patient groups (both $p \geq 0.9$; Table 2).

As hypothesized, the topological architecture of the LD functional connectome demonstrated widespread alterations of large-scale networks in this disorder. The optimal modular decomposition of the group-averaged network in healthy individuals revealed the following community formation: two frontotemporoparietal communities (modules I and III) each with clear hemispheric prevalence (Fig. $1 A$, red and purple), a bilateral basal ganglia/cerebellar community (module IV, orange), and a bilateral prefrontal community (module II, green). Compared with healthy controls, LD patients showed pronounced changes in network community structure (Fig. $1 A$ ) and a $p d$ of 0.24 . Specifically, LD formed an additional fifth functional community (module $\mathrm{V}$, blue) by fragmenting the solitary basal ganglia/cerebellar community (module IV) seen in healthy subjects into two communities in patients. The additional module $\mathrm{V}$ in $\mathrm{LD}$ was left lateralized to the basal ganglia and thalamus (Fig. 1A), pointing to abnormal functional integration of these areas in the large-scale network.

Within the detected modules, alterations in hub formation between the mean network of healthy controls and LD patients were observed on several levels, including: (1) degradation of connector to provincial hubs, (2) dropout of regions from hub level to high-influence nodes or below, and (3) formation of new hubs in the LD network that were not present in the healthy network (Fig. 1B). Specifically, the functional connectome of healthy individuals was characterized by an approximately bal- anced representation of provincial and connector hubs (12/17, respectively), whereas hub formation in the LD network was shaped by a dominant presence of provincial hubs with only few connectors (26/6, respectively). These hubs established strong connections with high-influence nodes, which were underrepresented in LD patients compared with healthy individuals. The brain regions that were most prone to these changes included the primary sensorimotor and parietal cortices, cerebellum, and thalamus (Fig. 1C).

Most notably, left primary somatosensory cortex (area 3b, connector hub), primary motor cortex (area $4 \mathrm{a}$, provincial hub), and premotor cortex (area 6, connector hub) were hubs in healthy subjects, but were not even among high-influence nodes in LD patients, suggesting a pathophysiological functional degradation of these regions in LD. Particularly, area $3 b$ showed the most pronounced drop in nodal influence (degree: $-31 \%$, strength: $-29 \%$ ) that was indicative of marked impairment of sensorimotor processing and integration in LD patients. Similarly, the functional impact of bilateral superior parietal cortex, which was found to host hub regions in normal controls (bilateral area $7 \mathrm{~A}$, left area $5 \mathrm{~L}$ : connectors; right area 5L: provincial), was drastically lower in LD patients illustrated by a decrease in degree and strength of up to $16 \%$ and $14 \%$, respectively. Therefore, bilateral parietal area 5L failed to show even elevated nodal influence values in the LD network and left and right parietal areas 7A were downgraded to a high-influence node and a provincial hub, respectively.

Conversely, left posterior parietal cortex exhibited abnormally increased functional influence (up to $22 \%$ and $30 \%$ in degree and strength, respectively) in the LD connectome, upgrading these regions to provincial hubs, albeit with decreased connectivity in the right hemisphere compared with healthy individuals (areas 7P/7PC; degree: $-22 \%$, strength: $-24 \%$ ). A similar pattern was found for the cerebellum (right lobules VIIIa/b, vermis) and thalamus, the left parietal and bilateral temporal subdivisions of which played the role of connector and provincial hubs, respectively, in the LD connectome, but were not classified as hubs in healthy volunteers. Finally, nodal influence metrics of the left extrastriate cortex (ventral part of area hOC5), which has been implicated in lateralization of language perception (Selpien et al., 2015) and was a network-wide relay in the healthy speech connectome, dropped by $20 \%$ (degree: $-20 \%$, strength: $-21 \%$ ) in LD patients, rendering its importance below high-influence nodes.

In summary, the loss of network-wide hub relays in left premotor, primary motor, somatosensory, and parietal regions, together with the emergence of connector hubs in the thalamus as part of the formation of a segregated nodal community in LD points to deficient global functional coupling of principle motor control areas accompanied by abnormal overrepresentation of thalamic activity in the LD functional network.

\section{Group 2: influence of genotype on the LD functional connectome}

A comparative analysis of functional networks constructed based on familial versus sporadic LD patients yielded no statistically significant differences in either nodal influence (all $p>0.7$ ) or global efficiency (all $p>0.2$ ) and no pronounced deviations in clustering coefficients (all $p>0.8$; Table 2). However, a more detailed analysis of graph segregation and integration based on the community structure of the respective group-averaged networks revealed visible alterations in network topologies across 
A

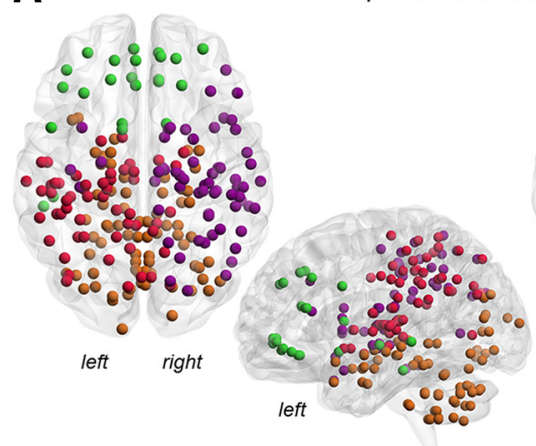

B

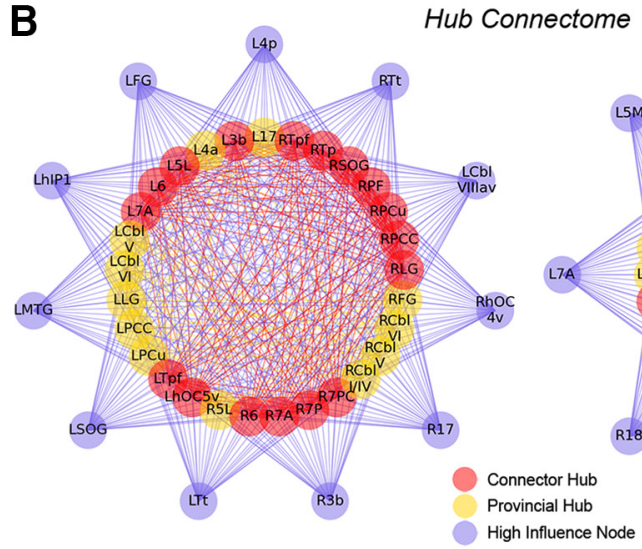

Healthy Volunteers

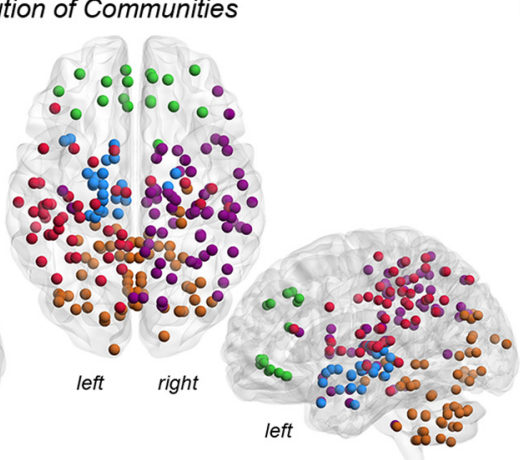

C

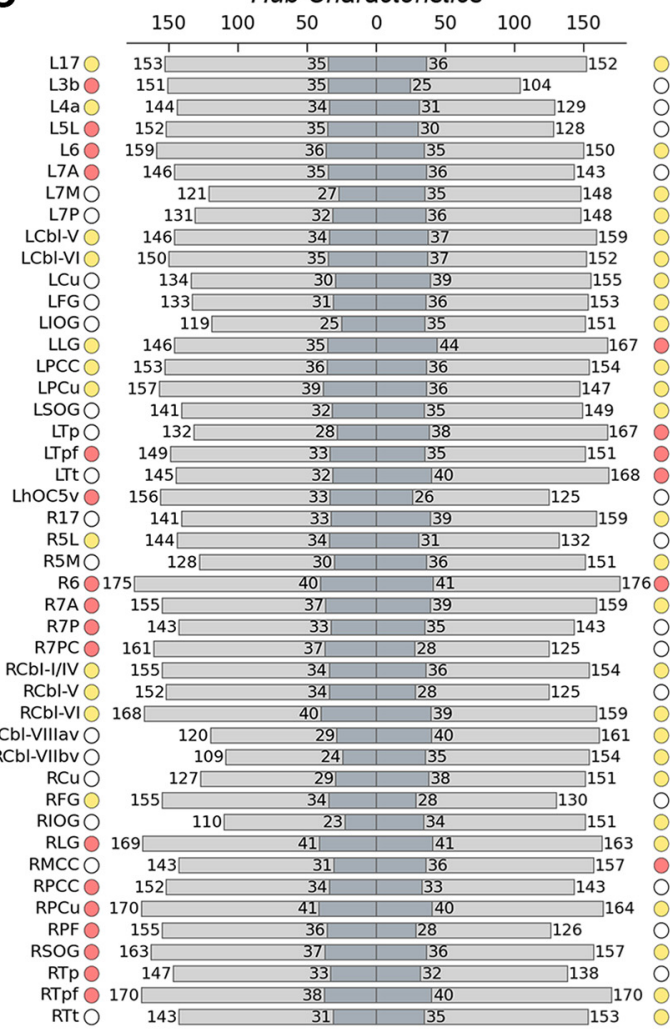

Healthy Volunteers General LD

Figure 1. Functional architecture of the group-averaged LD network compared with healthy individuals. $A$, Spatial topology of detected functional communities in the group-averaged healthy control (left) and LD patient network (right) is shown on 3D brain renderings in the axial and sagittal views, where regional module affiliation is illustrated by nodal color. $\boldsymbol{B}$, Bivariate (with respect to strength and degree) provincial (yellow) and connector hubs (red) and their connectivity profile with high-influence nodes (purple; nodes with degree and strength in the top $25 \%$ of the network) in the group-averaged healthy volunteer network (left) and in the mean LD graph (right). C, Visualization of variations in degree (light gray) and strength (dark gray) across provincial (yellow) and connector hubs (red) in the group-averaged healthy control and LD patient networks (left and right, respectively). White circles indicate nodes that did not qualify as hubs in the respective graph. 3b/6/17, Areas 3b/6/17; 4a, anterior part of area 4;5L/5M, subdivisions of area 5;7A/7M/7P/7PC, subdivisions of area 7; Cbl-I/IV/Cbl-V/Cbl-VI/Cbl-VIIlav/Cbl-VIIbbv, cerebellar lobules I/IV/V/VI/VIIla vermis; Cu/PCu, cuneus/precuneus; $\mathrm{FG}$, fusiform gyrus; IOG, inferior occipital gyrus; $\mathrm{LG}$, lingual gyrus; $\mathrm{MCC} / \mathrm{PCC}$, middle and posterior cingulate cortex; $\mathrm{PF}$, area PF in the inferior parietal cortex; SOG, superior occipital gyrus; $\mathrm{Tp} / \mathrm{Tpf} / \mathrm{Tt}$, parietal/prefronta/temporal subdivisions of the thalamus; $\mathrm{hOC5V}$, ventral part of area $\mathrm{hOC5}$; L, left; $\mathrm{R}$, right.

genotypes (Figs. 2, 3; sporadic vs familial cases with mixed heterogeneous ADLD + ABLD phenotype: $p d=0.22$ or ADLD-only homogeneous phenotype: $p d=0.25$ ).

The partitions of both sporadic networks showed a similar spatial distribution across phenotypes and a partition distance of $p d=0.17$ (heterogeneous vs homogeneous). Both heterogeneous and homogeneous sporadic LD networks comprised four communities (Figs. 2A, 3A) with a spatial topology comparable to the modules found in healthy controls (Fig. 1A). Sporadic LD networks were characterized by a large central module that contained $38 \%$ and $40 \%$ of all nodes in the graph across phenotypes and comprised all cerebellar and most basal ganglia and thalamic regions (Figs. $2 A, 3 A$, orange).

The spatial architecture of communities in familial networks was also consistent across phenotypes and exhibited a partition distance of $p d=0.14$ (heterogeneous vs homogeneous). Both heterogeneous and homogeneous familial LD networks were decomposed into five functional communities (Figs. $2 \mathrm{~A}, 3 \mathrm{~A}$ ), which resembled the partitioning of the composite LD patient network (Fig. 1A). The left basal ganglia thalamic and cerebellar regions formed a separate community (module V, blue) in familial LD networks independent of phenotype. These findings suggest different mechanisms by which subcortical areas are functionally integrated in familial versus sporadic LD neural networks.

\section{Influence of phenotype associations on the functional connectome of LD genotypes}

Hub connectivity in the familial network with heterogeneous phenotype was characterized by a high number of connectors vs provincial relays (22/7, respectively), which was contrasted by only three connectors out of a total of 25 hubs in the respective sporadic network (Fig. 2B). Similarly, the mixed-phenotype familial network recruited 19 high-influence nodes, whereas the mixed-phenotype sporadic graph only contained eight high-influence regions. Despite the fact that neither strength nor degree values showed pronounced differences between the two putative LD genotypes (with heterogeneous phenotype), the formation of hubs exhibited distinct deviations between the networks. Specifically, module V (Fig. 2B, blue) was one of the smallest nodal communities detected within the familial graph (comprising $9 \%$ of all nodes), but it contained five connector hubs. In contrast, module II (green) represented a community of the same size but did not contain any hubs. Notably, four of five connectors found in module $\mathrm{V}$ were left thalamic regions (motor, parietal, somatosensory, and temporal subdivisions), three of which (motor, parietal, and somatosensory thalamic subdivisions) failed to show elevated nodal influence levels in the sporadic network (Fig. 2C).

In contrast to the mixed-phenotype familial network, the familial ADLD-only group showed a prevalence of provincial hubs 
A

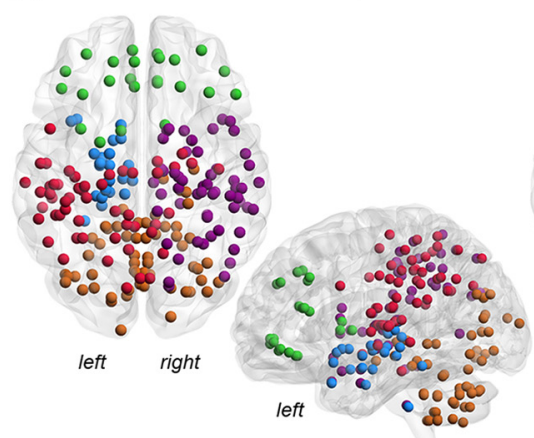

Spatial Distribution of Communities

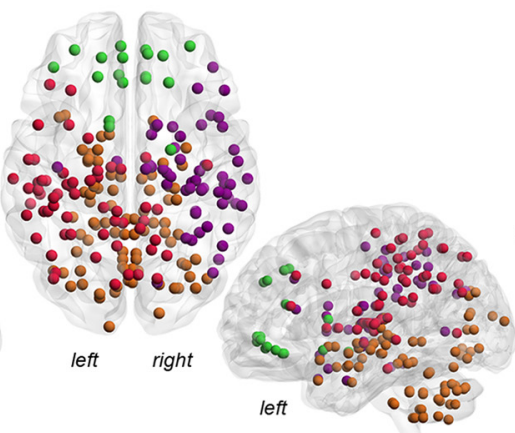

B

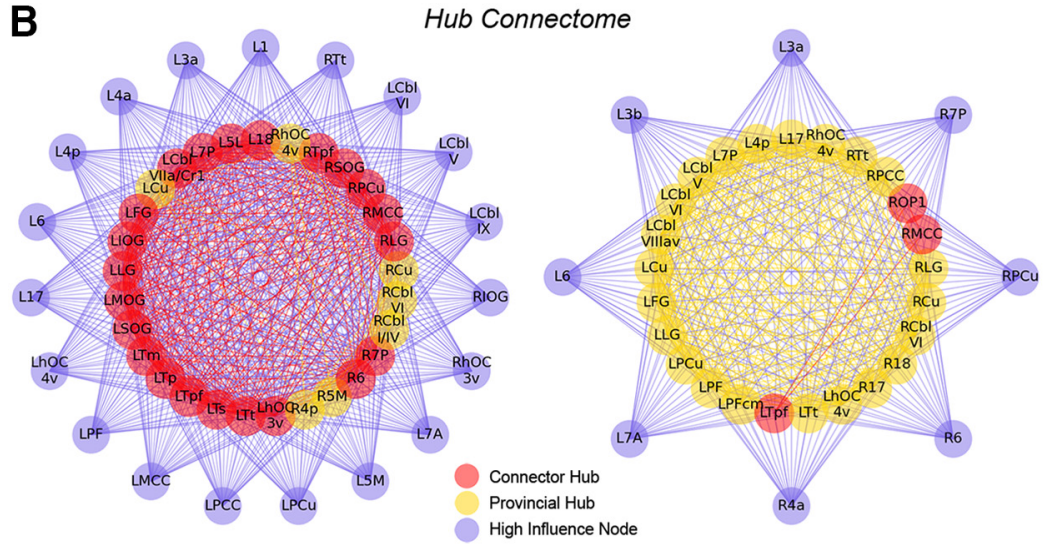

Familial LD (abductor+adductor)

Sporadic LD (abductor+adductor)

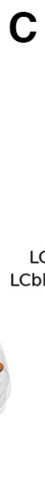

C

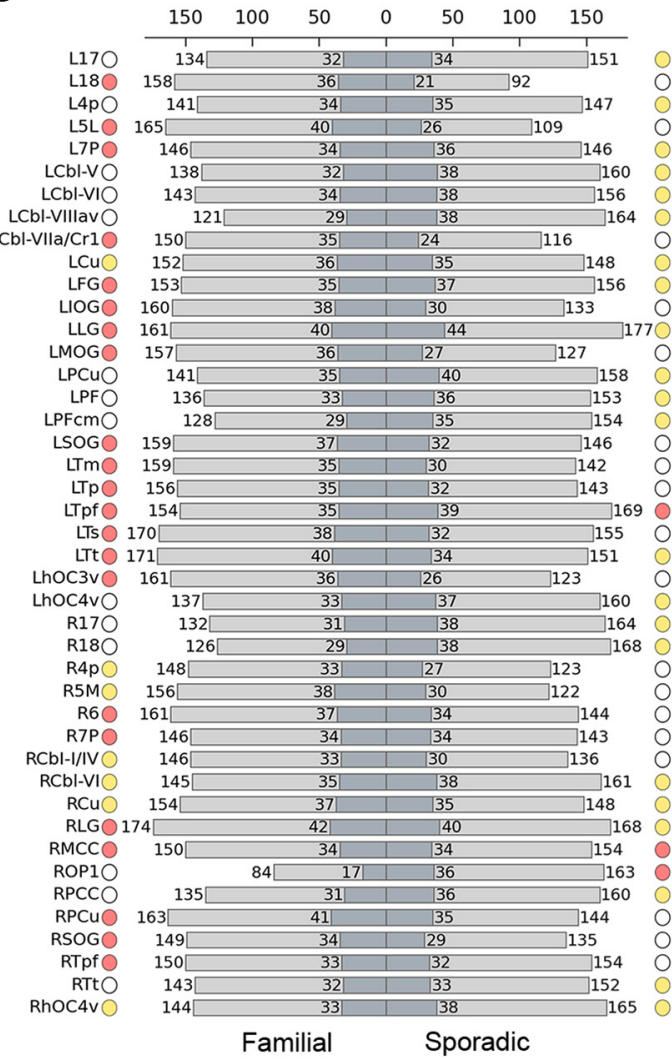

Familial Sporadic

Figure 2. Functional architecture of the group-averaged familial LD and sporadic LD networks with mixed phenotype. A, Spatial topology of detected functional communities in the groupaveraged mixed-phenotype familial LD (left) and sporadic LD networks (right) is shown on $3 D$ brain renderings in the axial and sagittal views, where regional module affiliation is illustrated by nodal color. B, Bivariate (with respect to strength and degree) provincial (yellow) and connector hubs (red) and their connectivity profile with high-influence nodes (purple; nodes with degree and strength in the top 25\% of the network) in the group-averaged mixed-phenotype familial LD (left) and in the mean mixed-phenotype sporadic LD graph (right). C, Visualization of variations in degree (light gray) and strength (dark gray) across provincial (yellow) and connector hubs (red) in the group-averaged mixed phenotype familial and sporadic LD networks (left and right, respectively). White circles indicate nodes that did not qualify as hubs in the respective graph. 6/17/18, areas 6/17/18;4p, posterior part of area 4;5L/5M/7P, subdivisions of parietal areas 5 and 7; $\mathrm{Cbl}-\mathrm{I} / \mathrm{IV} / \mathrm{Cbl}-\mathrm{V} / \mathrm{Cbl}$ $\mathrm{VI} / \mathrm{Cbl}-\mathrm{VIIlav} / \mathrm{Cbl}-\mathrm{VIIa} / \mathrm{Cr} 1$, cerebellar lobules I/IV/V/VI/VIIla vermis/VIla crus I; Cu/PCu, cuneus/precuneus; FG, fusiform gyrus; IOG/MOG/SOG, inferior/middle/superior orbital gyrus; LG, lingual gyrus; MCC/PCC, middle/posterior cingulate cortex; OP1, subdivision 1 of the operculum; PF/PFcm, areas PF/PFcm in the inferior parietal cortex; Tm/Tp/Tpf/Ts/Tt, motor/parietal/prefrontal/ somatosensory/temporal subdivisions of the thalamus; hOC3v/hOC $4 v$, ventral parts of areas $\mathrm{hOC} 3 / \mathrm{hOC} 4$; L, left; R, right.

with only a few connectors (connector/provincial: 4/25). This effect was also seen in the sporadic ADLD-only group, which exhibited only two connectors but 24 provincial hubs. Remarkably, module V (Fig. 3B, blue) played again a key role in the functional architecture of familial ADLD, containing two of four connectors (motor and parietal subdivisions of the left thalamus) and one provincial hub (somatosensory part of the left thalamus), while being the smallest nodal community in the network. Analogously to the mixed-phenotype networks, these three thalamic areas were not even considered high-influence nodes in the homogeneous sporadic group. Similarly, the familial ADLD network showed more high-influence nodes than the corresponding sporadic graph. These findings suggest that abnormal left thalamic function crucially shaped the familial LD connectome. Independent of phenotype, both familial networks showed an increase in high-influence nodes of $>36 \%$ compared with the corresponding sporadic graphs (Figs. 2B, 3B).

\section{Group 3: influence of phenotype on the LD functional connectome}

Network segregation estimated by the clustering coefficient showed pronounced differences between the group-averaged ABLD and ADLD networks independent of LD genotype (mixed heteroge- neous sporadic/familial genotypes vs homogeneous sporadic-only genotype: all $p<0.002$ ). Deviations in network integration between ADLD and ABLD graphs, which was approximated by the global efficiency, were not statistically significant for the networks with mixed-genotype ( $p=0.16)$, but exhibited a trend for sporadic-only graphs $(p=0.07)$. Both used nodal influence metrics were similar in the LD phenotype networks across examined genotypes (all $p>0.3$; Table 2).

These findings suggest that the two groups were characterized by distinctly different functional network architectures, which was confirmed by the computed optimal modular decompositions. Although sporadic ABLD and ADLD networks gave rise to four functional communities, the spatial distribution of these modules showed pronounced differences and a partition distance of $p d=0.26$. Notably, the modular architecture of the mixedgenotype ABLD network was characterized by the emergence of an additional fifth module and showed a higher degree of similarity to the mixed-genotype ADLD graph with a partition distance of $p d=0.2$. The difference in ABLD network architecture across genotypes was initially quantified by calculating the partition distance between mixed-genotype and sporadic-only ABLD networks $(p d=0.2)$. In contrast, the modular makeup of the 
A

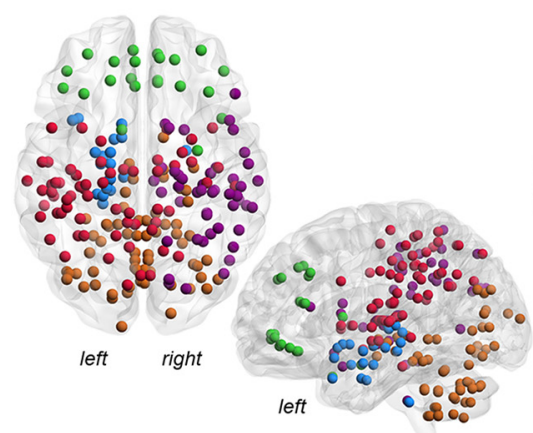

Spatial Distribution of Communities

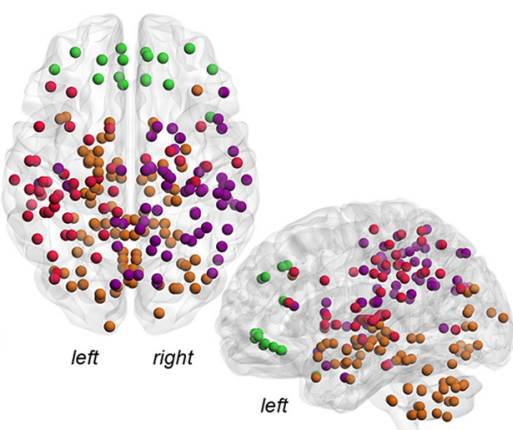

B

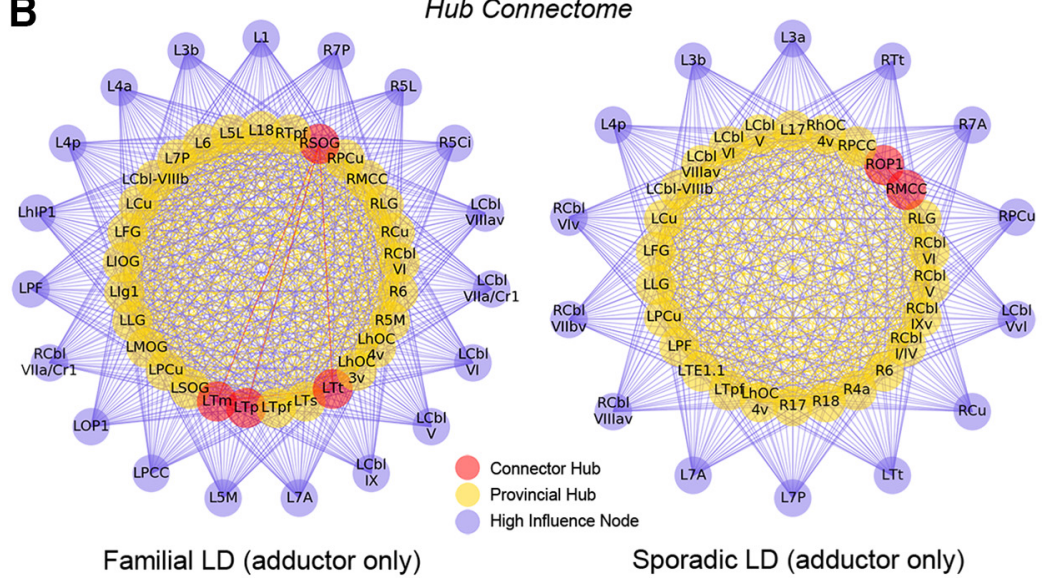

C

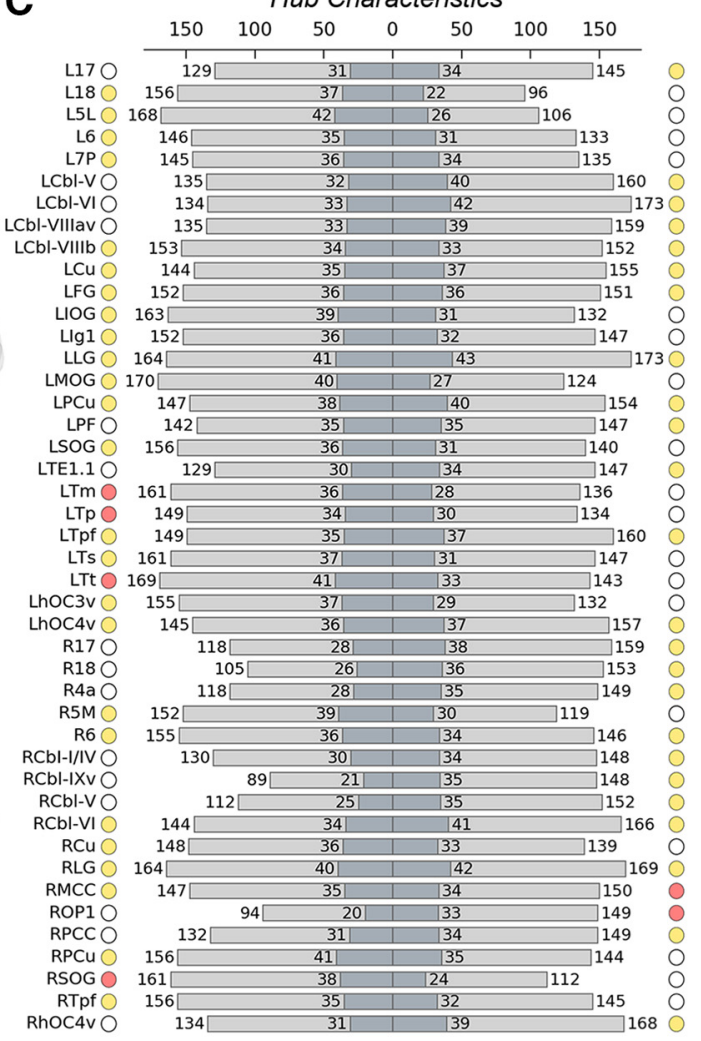

Familial Sporadic

Figure 3. Functional architecture of the group-averaged familial LD and sporadic LD networks with adductor phenotype. A, Spatial topology of detected functional communities in the group-averaged adductor familial LD (left) and adductor sporadic LD network (right) is shown on 3D brain renderings in the axial and sagittal views, where regional module affiliation is illustrated by nodal color. B, Bivariate (with respect to strength and degree) provincial (yellow) and connector hubs (red) and their connectivity profile with high-influence nodes (purple; nodes with degree and strength in the top $25 \%$ of the network) in the group-averaged adductor familial LD (left) and in the mean adductor sporadic LD graph (right). C, Visualization of variations in degree (light gray) and strength (dark gray) across provincial (yellow) and connector hubs (red) in the group-averaged adductor familial and adductor sporadic LD networks (left and right, respectively). White circles indicate nodes that did not qualify as hubs in the respective graph. 6/17/18, areas 6/17/18; 4a, anterior part of area 4; 5L/5M/7P, subdivisions of parietal areas 5/7; $\mathrm{Cbl}-\mathrm{I} / \mathrm{IV} / \mathrm{Cbl}-\mathrm{IXv} / \mathrm{Cbl}-\mathrm{V} / \mathrm{Cbl}-\mathrm{VI} /$ CbI-VIIIlav/Cbl-VIIIb, cerebellar lobules I/IV/IX vermis/V/VI/VIIla vermis/VIIIb; Cu/PCu, cuneus/precuneus; FG, fusiform gyrus; IOG/MOG/SOG, inferior/middle/superior occipital gyrus; Ig1, insula subdivision Ig1; LG, lingual gyrus; MCC/PCC, middle/posterior cingulate cortex; OP1, part 1 of the operculum; PF, parietal area PF; TE1.1, area TE1.1; Tm/Tp/Tpf/Ts/Tt, motor/parietal/prefrontal/ somatosensory/temporal subdivisions of the thalamus; h0C3v/hOC $4 v$, ventral parts of areas $\mathrm{hOC} 3 / \mathrm{hOC} 4$; L, left; R, right.

mixed vs homogeneous ADLD networks showed a high degree of spatial consistency and a partition distance of $p d=0.11$.

The modular structure of both networks exhibited characteristics similar to the partitioning of healthy individuals with two lateralized nodal communities (modules I, red, and III, purple), a large central group (module IV, orange), and a smaller frontal component (module II, green) (Figs. 4A, 5A). In contrast, the partitioning of the sporadic ABLD network showed little similarity to any other group. Most notably, module I (red) in the sporadic ABLD network was not restricted to the left hemisphere, but also included bilateral parietal regions, and module II (green) did not only comprise bilateral frontal, but also included left basal ganglia and temporal areas (Fig. 4A). The remaining two communities of the sporadic ABLD network (modules III, purple, and IV, orange) were similar to the corresponding nodal groups in sporadic ADLD patients. However, many of the nodes that were part of module III (purple) in the sporadic ADLD network were members of module I (red) in the sporadic ABLD graph, thus shrinking this right-lateralized community in sporadic ABLD compared with sporadic ADLD.

Conversely, the most notable characteristic of ABLD with mixed genotype was the emergence of a fifth functional module (blue) that was centered on left thalamic and basal ganglia regions
(Fig. 5A). Although this module was comparable to module $\mathrm{V}$ in the composite LD and mixed-phenotype familial LD networks (Figs. $1 A, 2 A$ ), it was smaller in size and restricted to the left hemisphere. Interestingly, this left-lateralized community almost exclusively comprised areas that were part of module II in the sporadic ABLD graph (Fig. $4 A$ ), with the exception of a single node (fascia dentata subdivision of the left hippocampus). Aside from this thalamic/basal ganglia community, the mixed-genotype ABLD graph showed a modular architecture similar to mixed-genotype ADLD that was shaped by two large clearly lateralized frontotemporoparietal communities (modules I, red, and III, purple; Fig. $5 \mathrm{~A}$ ), a bilateral prefrontal module (module II, green), and a bilateral basal ganglia/cerebellar community (module IV, orange). However, most basal ganglia regions that were part of module IV in the mixedgenotype ADLD network migrated to modules V and III in the left and right hemisphere, respectively, in mixed-genotype ABLD. Therefore, module IV was much smaller in the ABLD network with mixed genotype, comprising only $29 \%$ of nodes in contrast to $37 \%$ in mixed-genotype ADLD.

In summary, the observed variations in the modular architecture of ABLD networks across genotypes suggest an interplay of ABLD phenotype with familial genotype, which appeared to be a crucial driver for community formation in ABLD but not ADLD 
A

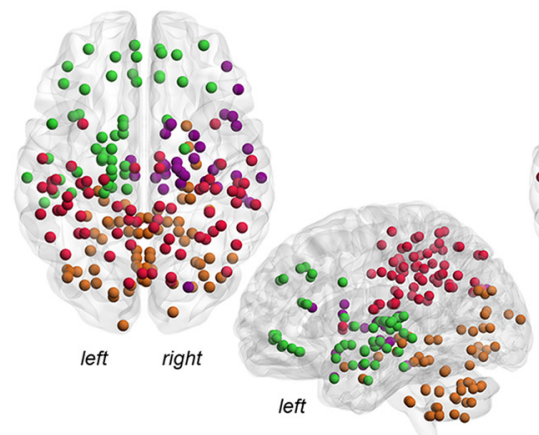

Spatial Distribution of Communities

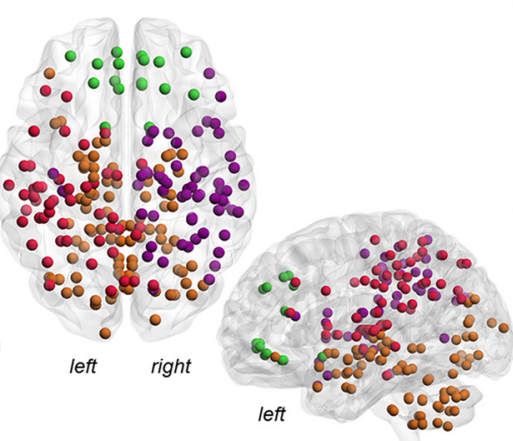

B

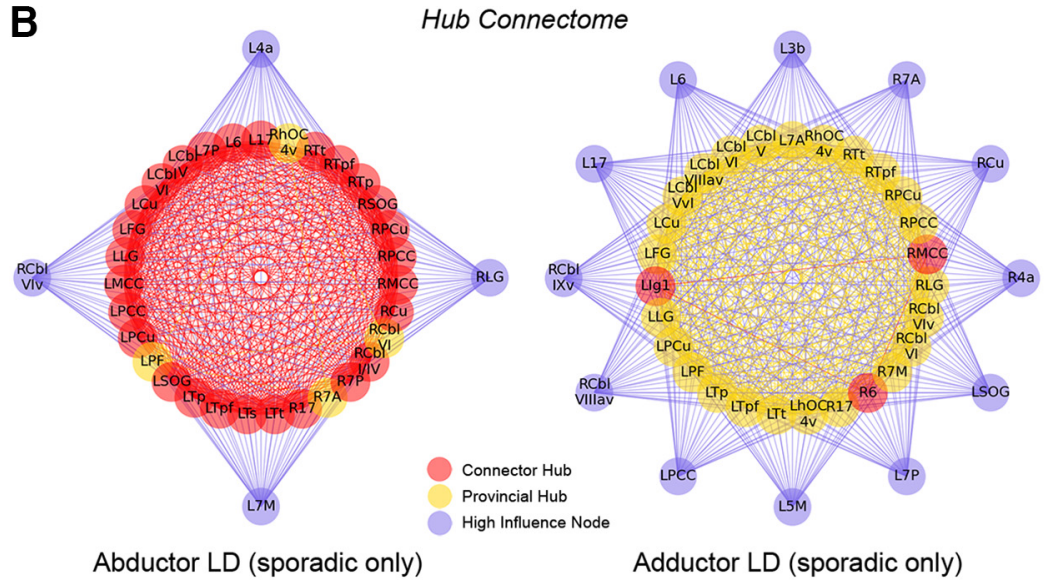

C

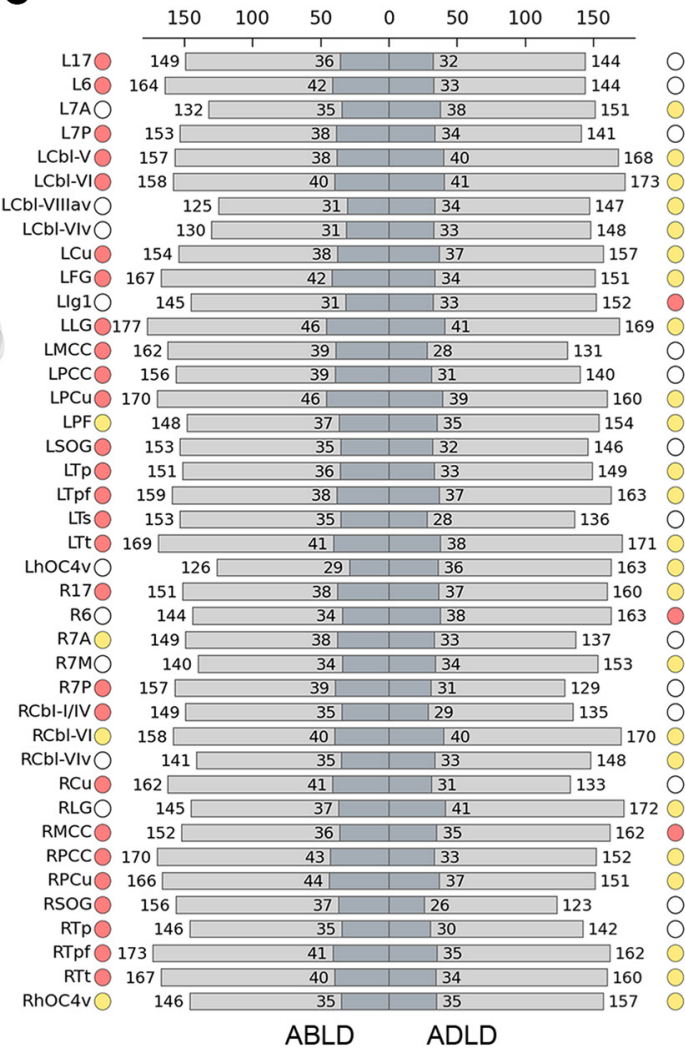

Figure 4. Functional architecture of the group-averaged abductor LD and adductor LD networks with sporadic genotype. $A$, Spatial topology of detected functional communities in the group-averaged abductor LD (left) and adductor LD networks with sporadic genotype (right) is shown on 3D brain renderings in the axial and sagittal views, where regional module affiliation is illustrated by nodal color. $\boldsymbol{B}$, Bivariate (with respect to strength and degree) provincial (yellow) and connector hubs (red) and their connectivity profile with high-influence nodes (purple; nodes with degree and strength in the top 25\% of the network) in the group-averaged abductor LD (left) and in the mean adductor LD graph with sporadic genotype (right). C, Visualization of variations in degree (light gray) and strength (dark gray) across provincial (yellow) and connector hubs (red) in the group-averaged sporadic abductor and adductor LD networks (left and right, respectively). White circles indicate nodes that did not qualify as hubs in the respective graph. 6/17, area 6/17; 7A/7M/7P, subdivisions of area 7; Cbl-I/IV/Cbl-V/Cbl-VI/Cbl-VIllav/Cbl-VIv, cerebellar lobules I/IV/V/VI/VIIla vermis/VI vermis; $\mathrm{Cu} / \mathrm{PCu}$, cuneus/precuneus; FG, fusiform gyrus; Ig1, part Ig1 of the insula; LG, lingual gyrus; MCC/PCC, middle/posterior cingulate cortex; PF, parietal area PF; SOG, superior orbital gyrus; Tp/Tpf/Ts/Tt, parietal/prefrontal/somatosensory/temporal subdivisions of the thalamus; hOC $4 v$, ventral part of area hOC4; L, left; R, right.

patients. Conversely, the latter showed a remarkably stable community architecture across genotypes.

\section{Influence of genotype associations on the functional connectome of LD phenotypes}

Hub formation varied markedly between sporadic ADLD and sporadic ABLD networks. Sporadic ADLD patients exhibited predominantly provincial hubs (provincial/connector: 24/3), whereas ABLD patients were characterized by a prevalence of connectors (provincial/connector: 4/27) (Fig. 4B). Similarly, the number of high-influence nodes was very low in the sporadic ABLD compared with the corresponding ADLD network (ABLD/ADLD: 4/12). Most notably, the left insula (subdivision Ig1) and the right premotor cortex (area 6) represented two out of a total of three connector hubs in the sporadic ADLD network, but failed to show any pronounced increase in nodal influence in the corresponding ABLD network (Fig. 4B).

Comparing LD phenotype networks with mixed genotypes revealed a similar pattern. Mixed-genotype ABLD showed twice as many connector hubs as the corresponding ADLD network ( 8 vs 4 ), whereas the number of high-influence nodes was much larger in mixed-genotype ADLD than in the ABLD graph ( 15 vs 2; Fig. 5B). Similarly, the same areas (left insular subdivision Ig1 and right area 6) represented provincial and connector hubs, respectively, in the mixed-genotype ADLD network but were not even classified as a high-influence node (insula) or downgraded to a provincial hub (area 6) for ABLD with mixed genotype (Fig. 5C).

Together, these findings point to a markedly different functional connectome of ABLD compared with ADLD with the ABLD network showing a distinct functional architecture dominated by a strongly coupled connector hub network spanning several nodal communities.

\section{Discussion}

In this study, we defined the functional connectome of the laryngeal form of isolated focal dystonia by means of an in-depth graph theoretical analysis of functional networks during symptomatic speech production. In Group 1, we identified that, in general, the LD functional connectome is shaped by abnormal functional integration of basal ganglia, cerebellum, and thalamus, as well as by pronounced functional deficiency of parietal and primary sensorimotor cortices. In Group 2, we demonstrated a driving role of left thalamus in forging the functional architecture of familial LD. In Group 3, we showed that the hub connectome of ABLD was dominated by connectors, whereas a prevalence of provincial hubs was a characteristic feature of ADLD independent of $L D$ genotype. The interplay of familial LD genotype with ADLD phenotype consistently resulted in five nodal communities by segregating left thalamic/basal ganglia regions, whereas the combination 
A

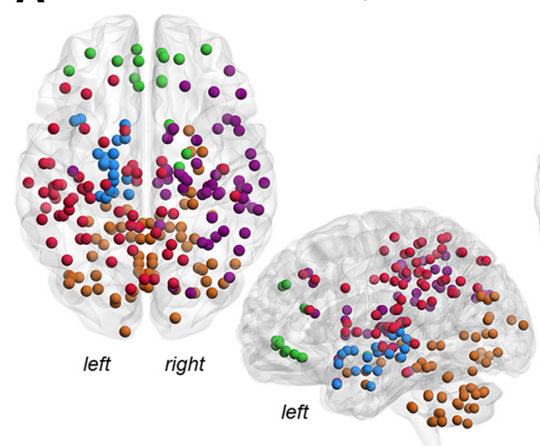

B

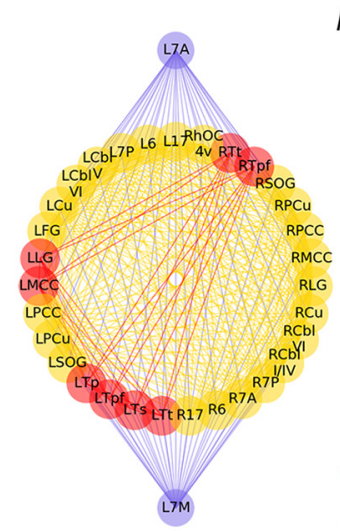

Hub Connectome

Abductor LD (sporadic+familial)

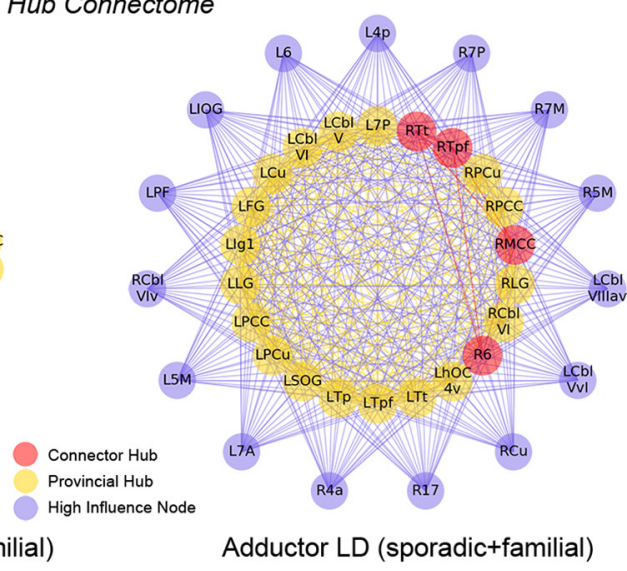

C

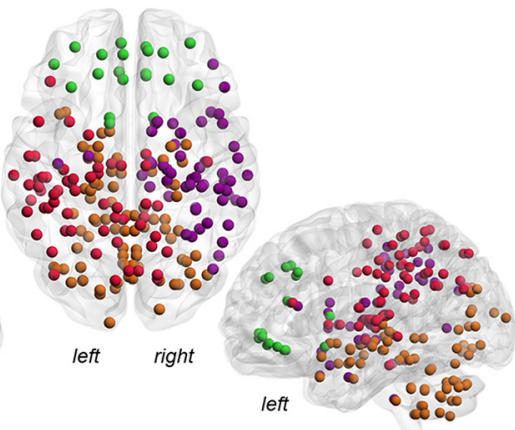

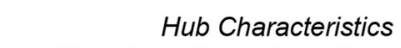

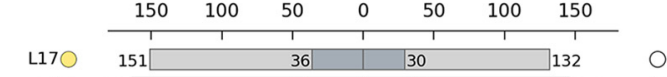

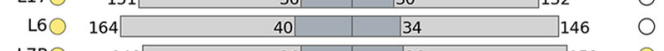

\begin{tabular}{ll|l|l|l|l|l|l|}
\hline L7PO 148 & 362 & 152
\end{tabular}

LCbl-VO 154 \begin{tabular}{|r|r|r|}
\hline & 37 & 41 \\
\hline
\end{tabular} $172 \bigcirc$

\begin{tabular}{ll|l|l|}
\hline LCbI-VIO & $1 6 0 \longdiv { 3 9 }$ & 42 & 179
\end{tabular}

\begin{tabular}{ll|l|} 
LCUO $1 5 8 \longdiv { 3 8 }$ & 38 \\
\hline
\end{tabular}

LFGO 168 \begin{tabular}{|r|r|}
\hline 41 & 35 \\
\hline
\end{tabular}

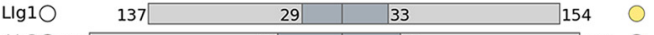

\begin{tabular}{r|c|c|}
\hline LLGO179 & 41 \\
\hline 39 & 30 & 0
\end{tabular}

\begin{tabular}{rr|r|}
\hline LPCC & $1 5 8 \longdiv { 1 3 9 }$ & 39 \\
\hline
\end{tabular}

\begin{tabular}{rr|r|r|}
\hline LPCuO 161 & 32 & 39 \\
\hline
\end{tabular}

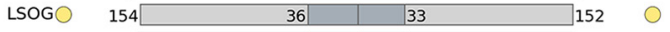

\begin{tabular}{l|l|l|l|}
\hline LTp 156 & 36 & 33 & 152
\end{tabular}

LTpfO 156 \begin{tabular}{|r|r|r|}
\hline 37 & 38 \\
\hline
\end{tabular}

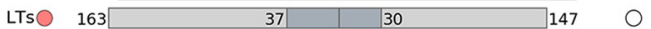

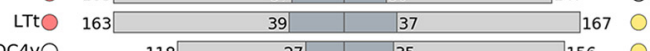

Lhoc4vO $\quad 118 \lcm{\square} 156$

\begin{tabular}{rr|r|r|r|}
\hline R17 157 & 32 & 141
\end{tabular}

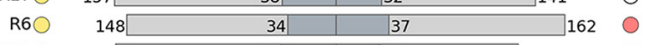

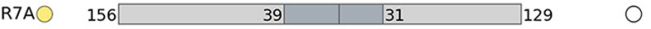

\begin{tabular}{rl|l|l|}
$\mathrm{R} 7 \mathrm{P} \bigcirc \quad 155$ & 38 & 135 & $\mathrm{O}$
\end{tabular}

RCbI-I/IVO 157 \begin{tabular}{|l|l|l|}
\hline 36 & 30 & 30 \\
\hline
\end{tabular}

RCbI-VIO 160 \begin{tabular}{r|r|r|}
\hline 406 & 39
\end{tabular}

\begin{tabular}{rr|r|r|}
$\mathrm{RCUO}$ & 159 \\
\hline & 40 & 33 & 344 \\
\hline
\end{tabular}

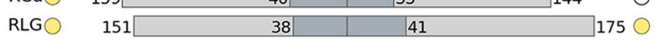

\begin{tabular}{rr|r|r} 
RMCC $\bigcirc 1 5 6 \longdiv { 3 6 }$ & 35 & 156
\end{tabular}

\begin{tabular}{r|r|r|} 
RPCC $1 6 6 \longdiv { 4 1 }$ & 33 \\
\hline
\end{tabular}

RPCu $1 6 7 \longdiv { \square } \begin{array} { r | c | c | } { \hline 4 3 } & { 3 8 } \\ { \hline } \end{array}$

RSOG 150 \begin{tabular}{r|r|r|}
\hline & 35 & 29 \\
140 & 39
\end{tabular}

\begin{tabular}{r|r|r|} 
RTpf & 167 \\
\hline & 39 & 35
\end{tabular}

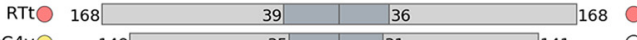

RhOC4v $\bigcirc$

148

ABLD

$$
\begin{aligned}
& 0 \\
& 0 \\
& 0 \\
& 0 \\
& 0 \\
& 0 \\
& 0 \\
& 0 \\
& 0 \\
& 0 \\
& 0 \\
& 0 \\
& 0 \\
& 0 \\
& 0 \\
& 0 \\
& 0 \\
& 0
\end{aligned}
$$

31

Figure 5. Functional architecture of the group-averaged abductor LD and adductor LD networks with mixed genotype. $\boldsymbol{A}$, Spatial topology of detected functional communities in the groupaveraged abductor LD (left) and adductor LD networks with mixed genotype (right) is shown on 3D brain renderings in the axial and sagittal views, where regional module affiliation is illustrated by nodal color. $\boldsymbol{B}$, Bivariate (with respect to strength and degree) provincial (yellow) and connector hubs (red) and their connectivity profile with high-influence nodes (purple; nodes with degree and strength in the top $25 \%$ of the network) in the group-averaged abductor LD (left) and in the mean adductor LD graph with mixed genotype (right). $C$, Visualization of variations in degree (light gray) and strength (dark gray) across provincial (yellow) and connector hubs (red) in the group-averaged mixed-genotype abductor and adductor LD networks (left and right, respectively). White circles indicate nodes that did not qualify as hubs in the respective graph. 6/17, area 6/17;7A/7P, subdivsions of area 7; Cbl-I/IV/Cbl-V/Cbl-VI, cerebellar lobules I/IV/V/Vl; Cu/PCU, cuneus/precuneus; FG, fusiform gyrus; Ig1, part Ig1 of the insula; LG, lingual gyrus; MCC/PCC, middle/posterior cingulate cortex; SOG, superior orbital gyrus; Tp/Tpf/Ts/Tt, parietal/prefrontal/somatosensory/temporal subdivisions of the thalamus; hOC $4 v$, ventral part of area hOC4; L, left; R, right.

of sporadic genotype and ADLD phenotype always yielded four communities. Therefore, at the level of functional networks, the association of LD genotypes and phenotypes was reflected by the interplay of community formation and intramodule vs intermodule hub configuration.

\section{Group 1: Overall characterization of the LD functional connectome}

The connectivity profile in LD patients differed from that in healthy individuals on two levels. First, a clearly delineated left thalamic/basal ganglia community played a crucial role in the functional architecture of LD network; second, the majority of LD hubs were local relays. The additional fifth functional community in LD was centered on left basal ganglia and thalamus and only comprised $12 \%$ of network nodes, although it contained two of the total six connector hubs, which were both left thalamic regions. This indicates that, in a dystonic network, the left thalamus acted as a transmitter that relayed network-wide information to left basal ganglia forming a closely interacting community. This finding is consistent with the results of earlier study that illustrated the segregation of basal ganglia, thalamic and cerebellar resting-state communities across different focal dystonia (Battistella et al., 2017). Considering that both basal ganglia and cerebellar networks converge in the motor cortex (Bostan and Strick, 2010), our results suggest that pathophysiological integra- tion of basal ganglia structures in the motor control network may propagate alterations via left thalamic structures throughout the entire network and likely contribute to the dystonic cascade. Together, these findings not only support previous reports of functional alterations in these regions in dystonia (Galardi et al., 1996; Neychev et al., 2008; Simonyan and Ludlow, 2012), but also reveal important details pertaining to the highly abnormal functional embedding of these key areas in the global large-scale brain network in LD.

A detailed assessment of hub structure showed a marked prevalence of local relays in the LD network, which was in stark contrast to a balanced distribution of hubs participating in long- and short-range edges in the healthy network. LD connectivity patterns exhibited a shift toward nodal cliques with strong intragroup connections but minimal network-wide relays, pointing to an abnormally segregated network architecture compared with healthy individuals. The latter was also reflected by significantly increased global clustering coefficient values in LD patients. Specifically, left primary motor and somatosensory cortices (areas $4 \mathrm{a}$ and $3 \mathrm{~b}$ ), which are known to drive the functional connectome of speech in healthy individuals (Fuertinger et al., 2015), failed to show significant nodal influence in the LD network. The loss of these areas as provincial and connector hubs points to severely impaired cortical sensorimotor processing and execution in LD. This also provides an explanation for abnormal sensorimotor 
alterations, particularly in task-specific focal dystonias (BaraJimenez et al., 2000; Fiorio et al., 2003; Tinazzi et al., 2006; Termsarasab et al., 2016). Similarly, left premotor cortex (area 6) was downgraded from a connector hub in the healthy network to a provincial relay in LD. Given its importance for sensorimotor integration, motor learning, and planning during speech production (Rauschecker, 2011; Jenson et al., 2014; Hardwick et al., 2015), altered function of this hub may additionally contribute to abnormal execution of motor commands in LD. Profound alterations of sensorimotor network components observed during selective impairment of speech production as a complex voluntary behavior point to a hierarchical mechanism underlying dystonic symptoms.

This hierarchy also pertains to the loss of pivotal regions of higher-order sensorimotor processing in the bilateral superior parietal cortex (areas 5L and 7A; Culham and Valyear, 2006; Brownsett and Wise, 2010; Shum et al., 2011; Sereno and Huang, 2014). Particularly, functional degradation of these areas may have further deteriorating effects, not only on general aspects of motor planning and execution, but also on coding of task-specific aspects of complex voluntary movement patterns (Rathelot et al., 2017) such as speech production, presumably via a direct access to the laryngeal motor cortex (Simonyan and Jürgens, 2002; Kumar et al., 2016). Conversely, increased functional influence of other parietal regions (left areas 7M and 7P, right area 5M), which were local functional relays in the dystonic network but were not even among the high-influence nodes in the healthy network, may point to a compensatory and abnormal upregulation of sensorimotor processing. Because recent studies suggested a potentially important role for parietal cortex in the pathophysiology of dystonia (Battistella et al., 2016; Gallea et al., 2016; Putzel et al., 2016a; Bianchi et al., 2017), our current findings provide more precise insights into abnormal functional embedding and specialized influences of the parietal cortex within the large-scale LD network.

\section{Group 2: influence of genotype on the LD functional connectome}

The functional architecture of familial LD networks was characterized by the formation of five nodal communities. Among these, a segregated community was centered on the basal ganglia and thalamus, where left thalamus acted as a global network relay station, functionally embedding the basal ganglia into the largescale brain network. Notably, the parietal thalamic subdivision was found to be a shared connector hub across all familial networks independent of LD phenotype. This points to further abnormal integration of the parietal cortex via its subcortical connectivity.

In contrast to familial LD, basal ganglia and thalamus did not form a separate community in sporadic $\mathrm{LD}$, but were rather integrated in a larger subcortical-cerebellar community. This network topology resembled that of the healthy network, possibly suggesting that the cerebellothalamocortical circuit was less impaired in the sporadic than familial LD. As another characteristic feature of the sporadic LD connectome, the hub distribution in both ADLD-only and mixed-phenotype sporadic LD networks was shaped by numerous provincial hubs and only a few connectors. This suggests that the pathophysiology underlying the combination of ADLD phenotype with sporadic genotype was characterized by a loss of network-wide hub relays in key areas of central motor control and sensorimotor processing.

\section{Group 3: influence of phenotype on the LD functional connectome}

Independent of genotype, the ADLD network exhibited a community structure similar to sporadic networks, whereas the ABLD network was shaped by highly distinct modular characteristics. Specifically, sporadic ABLD network was characterized by a large functional community that comprised $30 \%$ of all network nodes, including 10 of 27 connector hubs, and spanned bilateral superior parietal cortex. The latter was abnormally coupled with premotor, primary sensorimotor, and middle and posterior cingulate cortices. Altered functional connectivity profile of this nodal community suggests multilevel aberrations of sensory and motor transformations in the pathophysiology of dystonia due in part to substantial involvement of the parietal cortex as an important region of higher-level sensorimotor processing and integration.

Four connector hubs of the sporadic ABLD-only network, all of which were left thalamic areas (parietal, prefrontal, somatosensory, and temporal subdivisions), were embedded with basal ganglia and bilateral frontal regions in the same nodal community. This may suggest that the left thalamus plays a key role in sporadic ABLD hub connectome by establishing network-wide connections as part of frontothalamic nodal community. This network topology may underlie alterations of executive processing in LD such as abnormal sensory temporal discrimination (Termsarasab et al., 2016). It is also notable that the same thalamic regions (except for the prefrontal subdivision) were found to be connector hubs located in a segregated subcortical community of the mixed-genotype ABLD network, once again pointing to the function of this structure as a global relay station.

While the hub formation of sporadic ABLD network was dominated by connectors, sporadic ADLD was characterized by numerous provincial hub relays. The cerebellar frontoparietal community was the largest nodal group in the sporadic ADLD network that comprised $40 \%$ of all network nodes. It encompassed thalamus, cerebellum, cingulate, and parietal and frontal cortices, containing 16 of 24 provincial hubs. The formation of this large community, together with an overrepresentation of local intramodular hubs, might be associated with dystoniarelated cortical hyperexcitability because the cerebellum has been shown to establish projections with association cortex in prefrontal, parietal, and superior temporal areas (Kelly and Strick, 2003). Although the mixed-genotype ABLD network exhibited more connectors than corresponding ADLD network, its hub connectome was primarily shaped by provincial relays. Therefore, abnormally high connector hub formation was not just driven by the ABLD phenotype; the interplay of sporadic genotype with ABLD phenotype yielded excessive connector hub recruitment.

\section{Summary}

On a network level, LD genotype and phenotype associations were reflected by the interplay of community organization and the formation of intramodule vs intermodule relay nodes. LD was characterized by abnormal global functional coupling of sensorimotor cortical areas, particularly primary sensorimotor and parietal cortices, as well as cerebellum, basal ganglia, and thalamus. Our results point to left thalamus as a key driver of functional network aberrations and abnormal connector hub recruitment, as well as to altered connectivity of parietal cortex as an important hallmark of LD connectome. The combination of sporadic genotype with ADLD phenotype was associated with a connectome that was mainly populated by provincial hubs, whereas familial genotype with ABLD phenotype yielded numer- 
ous long-range connector hubs spread out over functional communities. The connectome-wide approach taken in this study may serve as a blueprint for similar studies in other forms of focal dystonia for the discovery of pathophysiological mechanisms underlying this disorder that are both form-specific and shared across dystonias.

\section{References}

Ali SO, Thomassen M, Schulz GM, Hosey LA, Varga M, Ludlow CL, Braun AR (2006) Alterations in CNS activity induced by botulinum toxin treatment in spasmodic dysphonia: an H215O PET study. J Speech Lang Hear Res 49:1127-1146. Medline

Bara-Jimenez W, Shelton P, Hallett M (2000) Spatial discrimination is abnormal in focal hand dystonia. Neurology 55:1869-1873. CrossRef Medline

Battistella G, Fuertinger S, Fleysher L, Ozelius LJ, Simonyan K (2016) Cortical sensorimotor alterations classify clinical phenotype and putative genotype of spasmodic dysphonia. Eur J Neurol 23:1517-1527. CrossRef Medline

Battistella G, Termsarasab P, Ramdhani RA, Fuertinger S, Simonyan K (2017) Isolated focal dystonia as a disorder of large-scale functional networks. Cereb Cortex 27:1203-1215. Medline

Bianchi S, Battistella G, Huddleston H, Scharf R, Fleysher L, Rumbach AF, Frucht SJ, Blitzer A, Ozelius LJ, Simonyan K (2017a) Phenotype- and genotype-specific structural alterations in spasmodic dysphonia. Mov Disord 32:560-568. CrossRef Medline

Bostan AC, Strick PL (2010) The cerebellum and basal ganglia are interconnected. Neuropsychol Rev 20:261-270. CrossRef Medline

Brillinger DR (2002) Second-order moments and mutual information in the analysis of time series. In: Recent advances in statistical methods: proceedings of Statistics 2001 Canada, the 4th Conference in Applied Statistics. Montreal, 6-8 July 2001 (Chaubey YP, ed), pp 64-76.

Brownsett SL, Wise RJ (2010) The contribution of the parietal lobes to speaking and writing. Cereb Cortex 20:517-523. CrossRef Medline

Bullmore E, Sporns O (2009) Complex brain networks: graph theoretical analysis of structural and functional systems. Nat Rev Neurosci 10:186198. CrossRef Medline

Cover TM, Thomas, J.A (1991) Elements of information theory. New York: Wiley.

Cox RW (1996) AFNI: software for analysis and visualisation of functional magnetic resonance neuroimages. Comput Biomed Res 29:162-173. CrossRef Medline

Culham JC, Valyear KF (2006) Human parietal cortex in action. Curr Opin Neurobiol 16:205-212. CrossRef Medline

Eickhoff SB, Stephan KE, Mohlberg H, Grefkes C, Fink GR, Amunts K, Zilles K (2005) A new SPM toolbox for combining probabilistic cytoarchitectonic maps and functional imaging data. Neuroimage 25:1325-1335. CrossRef Medline

Fakcharoenphol J, RaoS (2006) Planar graphs, negative weight edges, shortest paths, and near linear time. J Comput Syst Sci 72:868-889. CrossRef

Fiorio M, Tinazzi M, Bertolasi L, Aglioti SM (2003) Temporal processing of visuotactile and tactile stimuli in writer's cramp. Ann Neurol 53:630635. CrossRef Medline

Fortunato S (2010) Community detection in graphs. Phys Rep 486:75-174. CrossRef

Fuertinger S, Simonyan K (2016) Stability of network communities as a function of task complexity. J Cogn Neurosci 28:2030-2043. CrossRef Medline

Fuertinger S, Horwitz B, Simonyan K (2015) The functional connectome of speech control. PLoS Biol 13:e1002209. CrossRef Medline

Fuertinger S, Simonyan K, Sperling MR, Sharan AD, Hamzei-Sichani F (2016) High-frequency brain networks undergo modular breakdown during epileptic seizures. Epilepsia 57:1097-1108. CrossRef Medline

Galardi G, Perani D, Grassi F, Bressi S, Amadio S, Antoni M, Comi GC, Canal N, Fazio F (1996) Basal ganglia and thalamo-cortical hypermetabolism in patients with spasmodic torticollis. Acta Neurol Scand 94:172-176. CrossRef Medline

Gallea C, Horovitz SG, Ali Najee-Ullah M, Hallett M (2016) Impairment of a parieto-premotor network specialized for handwriting in writer's cramp. Hum Brain Mapp 37:4363-4375. CrossRef Medline

Hardwick RM, Lesage E, Eickhoff CR, Clos M, Fox P, Eickhoff SB (2015)
Multimodal connectivity of motor learning-related dorsal premotor cortex. Neuroimage 123:114-128. CrossRef Medline

Haslinger B, Erhard P, Dresel C, Castrop F, Roettinger M, Ceballos-Baumann AO (2005) "Silent event-related" fMRI reveals reduced sensorimotor activation in laryngeal dystonia. Neurology 65:1562-1569. CrossRef Medline

Humphries MD, Gurney K, Prescott TJ (2006) The brainstem reticular formation is a small-world, not scale-free, network. Proc Biol Sci 273:503511. CrossRef Medline

Hunter JD (2007) Matplotlib: A 2D graphics environment. Comput Sci Eng 9:90-95.

Jenson D, Bowers AL, Harkrider AW, Thornton D, Cuellar M, Saltuklaroglu $\mathrm{T}$ (2014) Temporal dynamics of sensorimotor integration in speech perception and production: independent component analysis of EEG data. Front Psychol 5:656. CrossRef Medline

Kelly RM, Strick PL (2003) Cerebellar loops with motor cortex and prefrontal cortex of a nonhuman primate. J Neurosci 23:8432-8444. Medline

Kinney JB, Atwal GS (2014) Equitability, mutual information, and the maximal information coefficient. Proc Natl Acad Sci U S A 111:3354-3359. CrossRef Medline

Kirke DN, Frucht SJ, Simonyan K (2015) Alcohol responsiveness in laryngeal dystonia: a survey study. J Neurol 262:1548-1556. CrossRef Medline

Kumar V, Croxson PL, Simonyan K (2016) Structural organization of the laryngeal motor cortical network and its implication for evolution of speech production. J Neurosci 36:4170-4181. CrossRef Medline

Latora V, Marchiori M (2001) Efficient behavior of small-world networks. Phys Rev Lett 87:198701. CrossRef Medline

Lynall ME, Bassett DS, Kerwin R, McKenna PJ, Kitzbichler M, Muller U, Bullmore E (2010) Functional connectivity and brain networks in schizophrenia. J Neurosci 30:9477-9487. CrossRef Medline

Meila M (2007) Comparing clusters: an information based distance. Journal of Multivariate Analysis 98:873-895. CrossRef

Newman ME (2006) Modularity and community structure in networks. Proc Natl Acad Sci U S A 103:8577-8582. CrossRef Medline

Neychev VK, Fan X, Mitev VI, Hess EJ, Jinnah HA (2008) The basal ganglia and cerebellum interact in the expression of dystonic movement. Brain 131:2499-2509. CrossRef Medline

Onnela JP, Saramäki J, Kertész J, Kaski K (2005) Intensity and coherence of motifs in weighted complex networks. Phys Rev E Stat Nonlin Soft Matter Phys 71:065103. CrossRef Medline

Perrachione TK, Ghosh SS (2013) Optimized design and analysis of sparsesampling FMRI experiments. Front Neurosci 7:55. CrossRef Medline

Pesarin F (2001) Multivariate permutation tests with applications in biostatistics. New York: Wiley.

Putzel G, Battistella G, Rumbach A, Ozelius L, Sabuncu M, Simonyan K (2016a) Polygenic risk of spasmodic dysphonia is associated with vulnerable sensorimotor connectivity. Cereb Cortex 1-9. CrossRef

Putzel GG, Fuchs T, Battistella G, Rubien-Thomas E, Frucht SJ, Blitzer A, Ozelius LJ, Simonyan K (2016b) GNAL mutation in isolated laryngeal dystonia. Mov Disord 31:750-755. CrossRef Medline

Rathelot JA, Dum RP, Strick PL (2017) Posterior parietal cortex contains a command apparatus for hand movements. Proc Natl Acad Sci U S A 114:4255-4260. CrossRef Medline

Rauschecker JP (2011) An expanded role for the dorsal auditory pathway in sensorimotor control and integration. Hear Res 271:16-25. CrossRef Medline

Rubinov M, Sporns O (2010) Complex network measures of brain connectivity: uses and interpretations. Neuroimage 52:1059-1069. CrossRef Medline

Samargia S, Schmidt R, Kimberley TJ (2016) Cortical silent period reveals differences between adductor spasmodic dysphonia and muscle tension dysphonia. Neurorehabil Neural Repair 30:221-232. CrossRef Medline

Selpien H, Siebert C, Genc E, Beste C, Faustmann PM, Güntürkün O, Ocklenburg S (2015) Left dominance for language perception starts in the extrastriate cortex: an ERP and sLORETA study. Behav Brain Res 291: 325-333. CrossRef Medline

Sereno MI, Huang RS (2014) Multisensory maps in parietal cortex. Curr Opin Neurobiol 24:39-46. CrossRef Medline

Shannon CE (1948) A mathematical theory of communication. AT\&T Tech J 27:623-656.

Shum M, Shiller DM, Baum SR, Gracco VL (2011) Sensorimotor integra- 
tion for speech motor learning involves the inferior parietal cortex. Eur J Neurosci 34:1817-1822. CrossRef Medline

Simonyan K, Jürgens U (2002) Cortico-cortical projections of the motorcortical larynx area in the rhesus monkey. Brain Res 949:23-31. CrossRef Medline

Simonyan K, Ludlow CL (2010) Abnormal activation of the primary somatosensory cortex in spasmodic dysphonia: an fMRI study. Cereb Cortex 20:2749-2759. CrossRef Medline

Simonyan K, Ludlow CL (2012) Abnormal structure-function relationship in spasmodic dysphonia. Cereb Cortex 22:417-425. CrossRef Medline

Simonyan K, Tovar-Moll F, Ostuni J, Hallett M, Kalasinsky VF, LewinSmith MR, Rushing EJ, Vortmeyer AO, Ludlow CL (2008) Focal white matter changes in spasmodic dysphonia: a combined diffusion tensor imaging and neuropathological study. Brain 131:447-459. CrossRef Medline

Simonyan K, Berman BD, Herscovitch P, Hallett M (2013) Abnormal striatal dopaminergic neurotransmission during rest and task production in spasmodic dysphonia. J Neurosci 33:14705-14714. CrossRef Medline

Sporns O (2011) Networks of the brain. Cambridge, MA: MIT.
Strehl A, Ghosh J (2002) Cluster ensembles - a knowledge reuse framework for combining multiple partitions. Journal of Machine Learning Research 3:583-617.

Sun Y, Danila B, Josic K, Bassler KE (2009) Improved community structure detection using a modified fine-tuning strategy. EPL 86:2.

Termsarasab P, Ramdhani RA, Battistella G, Rubien-Thomas E, Choy M, Farwell IM, Velickovic M, Blitzer A, Frucht SJ, Reilly RB, Hutchinson M, Ozelius LJ, Simonyan K (2016) Neural correlates of abnormal sensory discrimination in laryngeal dystonia. Neuroimage Clin 10:18-26. CrossRef Medline

Tinazzi M, Fiorio M, Stanzani C, Moretto G, Smania N, Fiaschi A, Bhatia KP, Rothwell JC (2006) Temporal discrimination of two passive movements in writer's cramp. Mov Disord 21:1131-1135. CrossRef Medline

van den Heuvel MP, Sporns O (2011) Rich-club organization of the human connectome. J Neurosci 31:15775-15786. CrossRef Medline

van der Walt S, Colbert SC, Varoquaux G (2011) The NumPy array: a structure for efficient numerical computation. Comput Sci Eng 13:22-30.

Xia M, Wang J, He Y (2013) BrainNet viewer: a network visualization tool for human brain connectomics. PLoS One 8:e68910. CrossRef Medline 